\title{
Host defense mechanisms induce genome instability in an opportunistic fungal pathogen
}

\author{
Amanda Smith ${ }^{1}$ and Meleah A. Hickman ${ }^{*}, 1,2$
}

${ }^{*}$ Corresponding author

${ }^{1} \mathrm{PhD}$ program in Genetic and Molecular Biology, Emory University

${ }^{2}$ Department of Biology, Emory University

Key words: Candida albicans, Caenorhabditis elegans, fungal genomics, innate immunity, Reactive oxygen species, genetics 


\begin{abstract}
The ability to generate genetic variation facilitates rapid adaptation in stressful environments. The opportunistic fungal pathogen Candida albicans frequently undergoes large-scale genomic changes, including aneuploidy and loss-of heterozygosity $(\mathrm{LOH})$, following exposure to physiological stressors and host environments. However, the specific host factors that induce $C$. albicans genome instability remains largely unknown. Here, we leveraged genetically-tractable nematode hosts to specifically investigate the innate immune components driving hostassociated $C$. albicans genome instability, which include host production of antimicrobial peptides (AMPs) and reactive oxygen species (ROS). C. albicans associated with wildtype, immunocompetent hosts carried multiple large-scale genomic changes including $\mathrm{LOH}$, whole chromosome, and segmental aneuploidies. In contrast, $C$. albicans associated with immunocompromised hosts deficient in AMPs or ROS production had reduced LOH frequencies and fewer, if any, additional genomic changes. We also found that $C$. albicans cap $1 \Delta \Delta$ strains deficient in ROS detoxification, were more susceptible to host-produced ROS genome instability compared to wildtype $C$. albicans. Further, genomic perturbations resulting from host-produced ROS are mitigated by the addition of antioxidants. Taken together, this work suggests that hostproduced ROS and AMPs induces genotypic plasticity in C. albicans which may facilitate rapid adaptation and lead to phenotypic changes.
\end{abstract}

\title{
Introduction
}

The opportunistic fungal pathogen, Candida albicans, is a leading cause in fungal bloodstream infections, and $40 \%$ of these infections result in mortality ${ }^{1}$. In addition to bloodstream infections, C. albicans causes non-lethal mucosal infections, including vaginal and oral candidiasis ${ }^{1}$. Despite its ability to cause infection, $C$. albicans is typically commensal and a component of the human microbiome ${ }^{2}$. The ability of $C$. albicans to cause infection is highly dependent on the host context, including high estrogen levels ${ }^{3}$, chronic stress ${ }^{4}$, antibiotic use ${ }^{5-}$ ${ }^{7}$, uncontrolled diabetes ${ }^{8,9}$, and immunosuppression ${ }^{10,11}$. In the absence of proper immune recognition, fungal proliferation is uncontrolled, leading to infection. However, despite having a fully functioning immune system, healthy individuals also experience $C$. albicans infections. This is often due to commensal isolates within an individual that become pathogenic rather than an infection from outside sources ${ }^{12}$. This transition may be facilitated by $C$. albicans phenotypic and genotypic heterogeneity. C. albicans genetic diversity within and among individuals is very high and often include numerous single nucleotide polymorphisms (SNPs) and loss of heterozygosity $(\mathrm{LOH})$ events ${ }^{13}$. This genetic variation may be a direct consequence of the stressors $C$. albicans encounters in the host which include immune stressors and other microbes. Recent work has demonstrated the host environment elevates genome instability in C. albicans similar to in vitro stressors ${ }^{14-18}$. However, the specific host components that generate this instability is largely unknown.

Several studies investigating the diversity of clinical $C$. albicans isolates found that genomic variation was quite common ${ }^{13}, 19$. This variation included polymorphisms, copy number variation, loss-of-heterozygosity $(\mathrm{LOH})$, and partial or whole chromosomal aneuploidies ${ }^{19}$. This suggests that the host environment rapidly generates genetic variation. Recent studies involving murine infection models have found that when exposed to different host niches, $C$. albicans has higher levels of genetic variation compared to in vitro ${ }^{14-17}$. Similar to clinical isolates, laboratory $C$. albicans often had large-scale genomic changes including aneuploidy and LOH following murine infection ${ }^{14-16}$. In addition to murine models, Caenorhabditis elegans has been used as a model to assess $C$. albicans genome stability and strains that differ in genetic background and ploidy have elevated host-associated genome instability ${ }^{17}$. It is clear that host environments 
drive genetic diversity in $C$. albicans, however, it is remains unclear what specific host components contribute to $C$. albicans genome instability.

In the host, $C$. albicans likely encounters many different stressors within the host environment, including the immune system which is designed to control and remove pathogens. The human immune system recognizes pathogens, like $C$. albicans through pathogen recognition receptors (PRRs) that detect the specific microbial chemical signatures called pathogen-associated molecular patterns (PAMPS) ${ }^{20}$. The main cells involved in the recognition of $C$. albicans include monocytes, macrophages, and neutrophils ${ }^{21}$. This recognition triggers a specific cellular response. For instance, $\beta$-glucan is primarily recognized through CR3 and Dectin-1 which induces the production of antimicrobial peptides (AMPs) and pro-inflammatory cytokines $^{22}$. AMPs inhibit microbial growth through a variety of methods, including disruption of the cell membrane, and halt DNA, RNA, and protein synthesis ${ }^{23}$. The release of AMPs and proinflammatory cytokines recruit other immune cells to the site of infection including neutrophils ${ }^{21}$, 22. Upon phagocytosis of $C$. albicans, neutrophils and macrophages produce an oxidative burst, which generates reactive oxygen species $(\mathrm{ROS})^{21,22}$. ROS cause cellular toxicity through structural changes to the DNA ${ }^{24}$ and in C. albicans generates double strand breaks (DSBs) ${ }^{25}$. Together, host-produced AMPs and ROS act in various ways, damaging the cell membrane, and causing DNA damage in order to kill $C$. albicans cells.

C. albicans has several mechanisms to mitigate damage from host-produced ROS and AMPs, including secretion of peptide effectors ${ }^{26}$, induction of the HOG1 stress response pathway, the RAD53 DNA damage checkpoint, and upregulation of the transcription factor cap1 $\mathrm{p}^{27}$. Cap1p activates antioxidant genes that break down ROS ${ }^{27}$. Despite these mechanisms, C. albicans has a higher frequency of loss-of-heterozygosity ${ }^{28}$ following exposure to hydrogen peroxide in vitro. Similarly, tetraploid $C$. albicans acquire DSBs and undergo ploidy reduction in response to $\mathrm{ROS}^{25}$. However, we still don't understand the impact of host-produced ROS or AMPs on C. albicans genome stability.

To specifically investigate the impact of host-produced ROS and AMPs on C. albicans genome stability we can utilize the nematode, Caenorhabditis elegans. C. elegans is an extremely powerful model for investigating C. albicans pathogenesis and genome stability. In addition to its rapid life cycle, easy maintenance, and large brood sizes, it has a highly conserved innate immune system which includes both AMPs and ROS ${ }^{29}$. In response to $C$. albicans infection, C. elegans produce a majority of AMPs through the mitogen-activated protein kinase (MAPK) signaling cascade ${ }^{30,31}$. This pathway includes SEK-1 (MAPKK), homologous to the MKK3/6 and MKK4 mammalian MAPKKs. Mutations in SEK-1 increase susceptibility to $C$. albicans as well as other microbial pathogens demonstrating its importance in defense ${ }^{32,33}$. In addition to AMPs, C. elegans produce ROS as another host defense, similar to human macrophages. ROS are produced in response to bacterial and fungal pathogens and is mediated by the dual oxidase BLI- $3^{34-37}$. However, when BLI-3 is mutated, C. elegans are more susceptible to $C$. albicans ${ }^{38}$.

Here we investigated the role host-produced AMPs and ROS play in generating genome instability in $C$. albicans using the model host $C$. elegans. We infected healthy and two different immunocompromised hosts with mutations in either sek-1 (AMP production) or bli-3 (ROS production) with $C$. albicans and subsequently measured $\mathrm{LOH}$ frequency and genome-wide changes with whole-genome sequencing. We found that healthy hosts elevate genome instability and generate greater genetic diversity in $C$. albicans compared to immunocompromised hosts. Furthermore, our results suggest that the dual oxidase, BLI-3 produces genome instability through the production of ROS. The genome instability caused by host-produced ROS can however be alleviated with the addition of antioxidants. Taken together, our results suggest that host innate immune pathways cause genome instability in $C$. albicans. The genome diversity generated inside healthy hosts likely provide $C$. albicans with the ability to quickly adapt to a changing and stressful host environment. Further, these genome-wide 
perturbations may be a way in which $C$. albicans transitions from commensal to pathogenic in immunocompetent hosts.

\section{Results}

Host defense pathways elevate C. albicans genome instability

We, and others, have shown that nematode and murine host environments induce $C$. albicans genome instability compared to in vitro ${ }^{14-18}$, but the specific host attributes driving genome instability have yet to be elucidated. Here, we tested whether components of host innate immune function contributed to host-associated genome instability by comparing the frequency of loss-of-heterozygosity ( $\mathrm{LOH}$ ) between $C$. albicans extracted from healthy and immunocompromised hosts (Fig. 1A). We used two different immunocompromised host genotypes, one carrying a deletion of sek-1 which cannot produce AMPs ${ }^{39}$, and another host genotype carrying a deletion of bli-3 which cannot produce ROS ${ }^{35}, 37$. Host-associated LOH frequencies were reduced in $C$. albicans extracted from both sek-1 and bli-3 hosts compared to C. albicans extracted from healthy hosts (Fig. 1B). Following host exposure, the relative frequency of $\mathrm{LOH}$ of both laboratory and clinical $C$. albicans was significantly lower in sek-1 and bli-3 hosts compared to wildtype (N2) hosts (Figs. 1B, S1A\&B). Our previous work demonstrated that the bloodstream strain was more unstable compared to the laboratory strain. Despite this higher baseline instability, we found a significantly lower LOH frequency of bloodstream C. albicans extracted from wildtype hosts compared to bli-3 hosts (Fig. S1B).

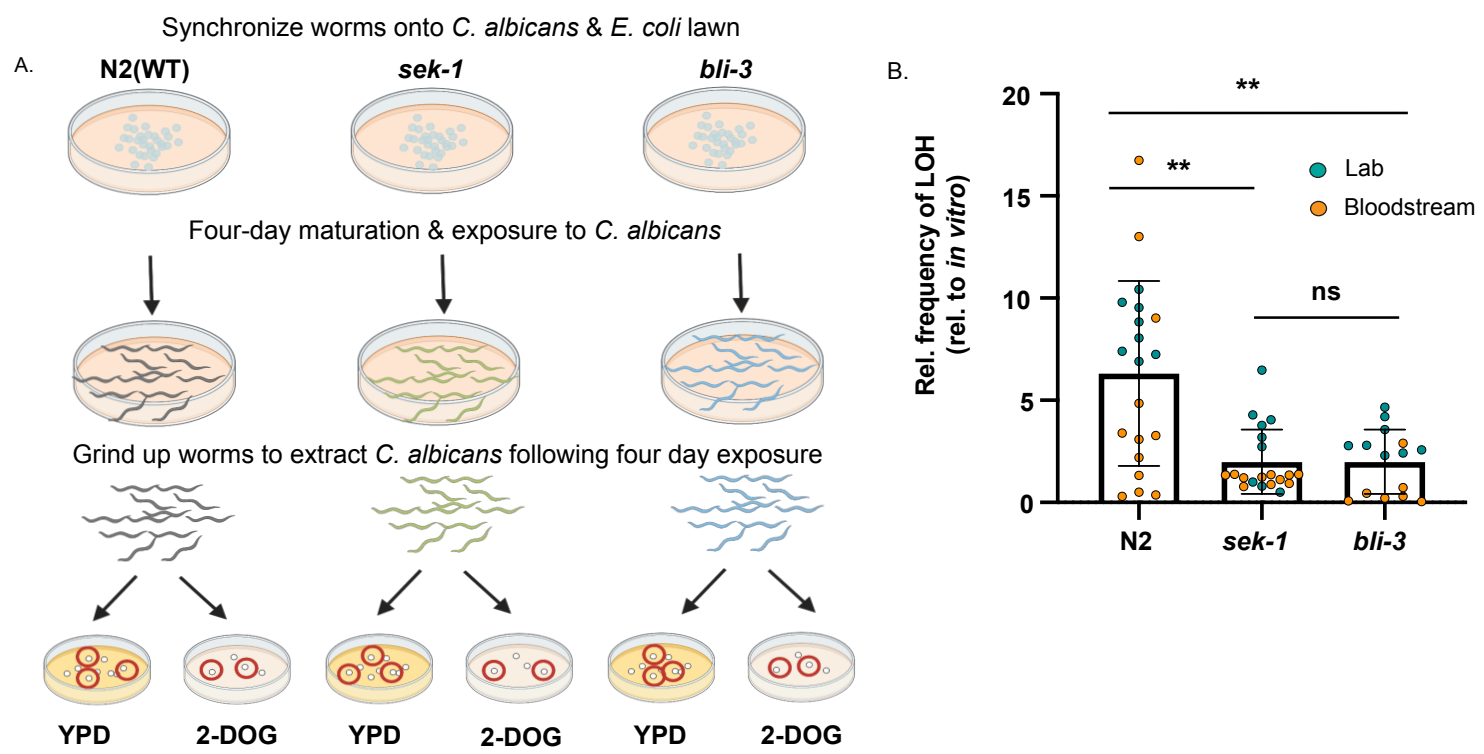

Randomly select colonies following host extraction for sequencing

Figure 1: Host immunity impacts $C$. albicans genome stability. A) Experimental overview of in vivo experiments. B) Laboratory (teal) and bloodstream (orange) C. albicans LOH frequency following host association relative to the LOH frequency of $C$. albicans no host control for N2, sek-1, and bli-3. Plotted are the means and SD. Asterisks indicate significant differences (**** $\mathrm{p}<$ 0.0001 , ns = not significant; Kruskal-Wallis with post-hoc Dunn's multiple test). 
Together, these data suggest that bli-3 and sek-1 immune pathways may be a source of genome instability in $C$. albicans regardless of pathogen genetic background.

\section{GAL1 LOH-selected isolates}

N2

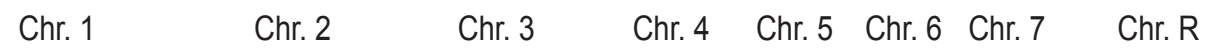

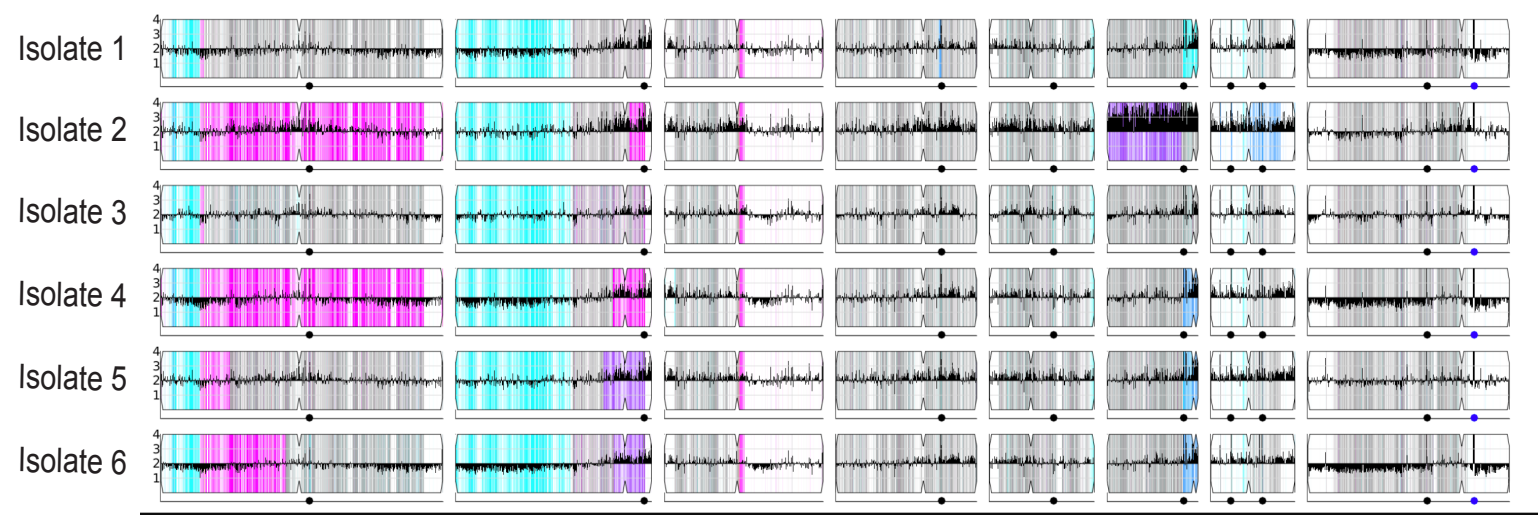

sek-1

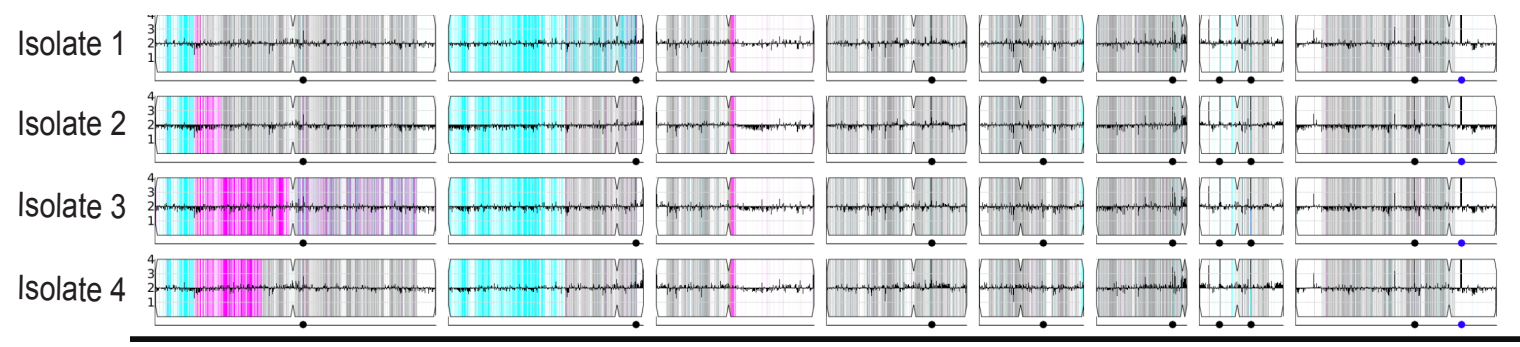

bli-3

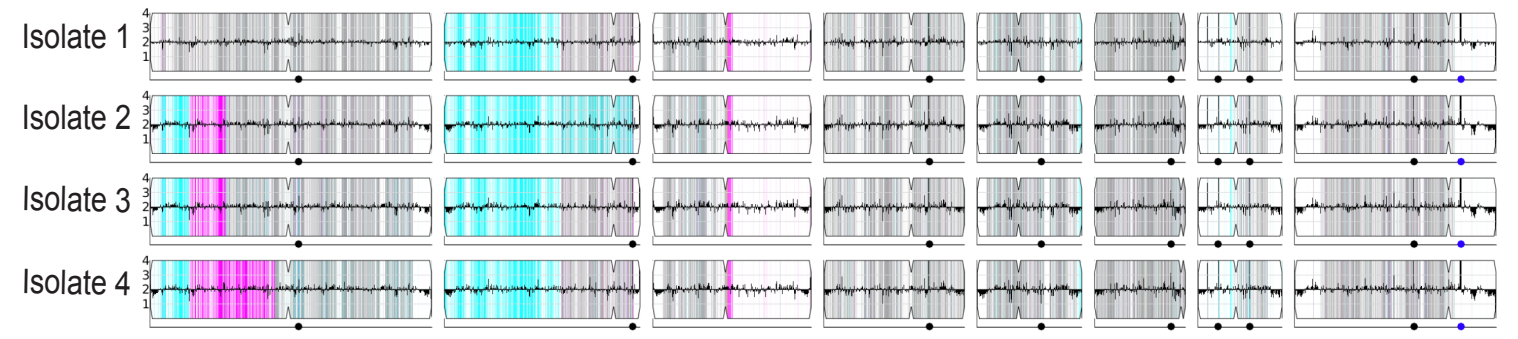

Figure 2: Genome-wide changes following host association.

YMAPs showing the copy number and allelic ratio for each GAL- isolate following host exposure.

Host immunity impacts the genomic landscape of C. albicans

While LOH assays are an easy and useful way to measure genome instability in $C$. albicans, they only provide a narrow focus into one small part of the genome. Therefore, we wanted to look more broadly and determine how host immunity impacts the genomic landscape of $C$. albicans. Following host-association and extraction from wildtype, sek-1 and bli-3 hosts we randomly selected single colonies from both YPD (no LOH selection) and 2-DOG (LOH, selection) and performed whole-genome sequencing. Large-tract LOH events and whole- 
chromosomal and segmental aneuploidy were frequently observed in $\mathrm{LOH}$-selected isolates from wildtype hosts, but not from either immunocompromised host background (Fig. 2). Whole or segmental aneuploidy was observed in $66 \%(4 / 6)$ the $\mathrm{LOH}$-selected isolates following wildtype host-association, but never from immunocompromised hosts. One isolate was trisomic for both chromosome 6 and 7 . Of the additional LOH tracts not selected for, all were caused by break-induced recombination. In contrast, we did not detect any aneuploidy in the isolates from either of the immunocompromised hosts (Fig. 2). We also investigated the impact of host exposure on the genomic landscape of isolates with no prior selection and found $50 \%(2 / 4)$ isolates had LOH events during exposure to healthy hosts (Fig. S2A). However, we did not detect any LOH events in the isolates extracted from immunocompromised hosts (Fig. S2A). Thus, healthy hosts induce more large-scale genomic changes in $C$. albicans regardless of $\mathrm{LOH}$ selection, compared to immunocompromised hosts.

We also investigated whether exposure to wildtype hosts generate more small-scale genetic changes in $C$. albicans compared to immunocompromised hosts. PCA analysis shows very little variation between the isolates, regardless of $\mathrm{LOH}$ selection or host background (Fig. $\mathrm{S} 2 \mathrm{~B}$ ), thus we grouped $\mathrm{LOH}$ selected and non- $\mathrm{LOH}$ selected isolates together for each host background and compared the number of SNPs and INDELS. Because ROS are mutagenic, we expected more mutations in C. albicans extracted from hosts and ROS. However, we detected no significant difference in the number of SNPs in the isolates extracted from the healthy or immunocompromised hosts (Fig. 3A). We did detect a significantly lower number of INDELs ( 1000 less) in isolates extracted from bli-3 hosts compared to sek-1 hosts (Fig. 3B). This suggests that the BLI-3 defense pathway generates INDELs in C. albicans. Despite incurring roughly the same number of mutations regardless of host background, larger chromosomal events including aneuploidy and large-scale LOH are primarily observed in isolates from wildtype hosts and not immunocompromised hosts.

A.

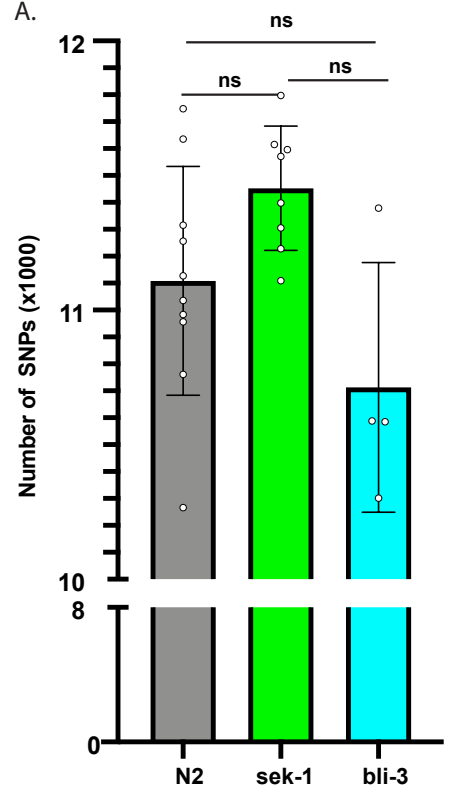

B.

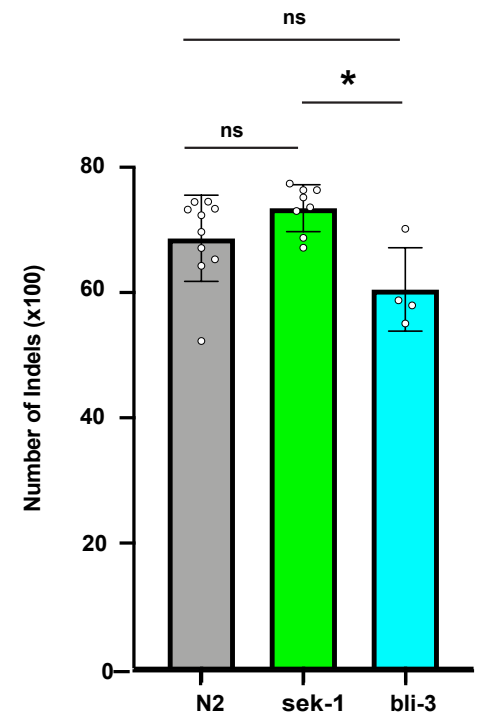

Figure 3: bli-3 hosts generate less genome-wide INDELs in $C$. albicans. A) Number of genomewide SNPs $(x 1000)$ in C. albicans following exposure to $\mathrm{N} 2$ (wildtype, grey, $\mathrm{n}=10$ ), sek-1 (green, $\mathrm{n}=8$ ), and bli-3 (blue, $\mathrm{n}=$ 4) hosts. Plotted are the mean and SD. Individual data points represent the number of SNPs in one isolate. B) Number of genome-wide INDELs $(x 100)$ in $C$. albicans following exposure to N2 (wildtype, grey, $n=10$ ), sek-1 (green, $\mathrm{n}=8$ ), and bli-3 (blue, $\mathrm{n}=$ 4) hosts. Plotted are the mean and SD. Individual data points represent the number of INDELs in one isolate. Asterisks indicate significant differences $\left({ }^{*} p<0.05\right.$, ns $=$ not significant; ANOVA with post-hoc Tukey's multiple test).

Investigating the role of ROS in generating genome instability 
Our data suggest that both sek-1 and bli-3 host immune pathways are a source of pathogen genome instability. While the SEK-1 MAPKK plays a central role in the MAPK cascade with many downstream targets, the main role of BLI-3 is generating ROS. Because of the clear connection between BLI-3 and ROS production, we wanted to investigate whether host-produced ROS is generating genome instability in $C$. albicans. We exposed both wildtype (SC5314) $C$. albicans and a cap1 $\Delta \Delta$ mutant strain of $C$. albicans deficient in detoxifying ROS, to $5 \mathrm{mM} \mathrm{H}_{2} \mathrm{O}_{2}$ for 24 hours in vitro and measured the frequency of $\mathrm{LOH}$. Similar to previous literature ${ }^{28}$, we found exposure to in vitro $\mathrm{H}_{2} \mathrm{O}_{2}$ increased $\mathrm{LOH}$ frequencies in both wildtype and cap $1 \Delta \Delta C$. albicans strains by 15 - and 40 -fold, respectively, when compared to the no stress treatment (Fig. 4A \& S3). We found that the relative $\mathrm{LOH}$ frequency following exposure to $\mathrm{H}_{2} \mathrm{O}_{2}$ was significantly higher in cap $1 \Delta \Delta C$. albicans compared to wildtype $C$. albicans. To directly test if host-produced ROS elevates $C$. albicans genome instability, we compared cap $1 \Delta \Delta C$. albicans $\mathrm{LOH}$ frequency to wildtype $C$. albicans $\mathrm{LOH}$ frequency following host exposure. If hostproduced ROS elevate $C$. albicans LOH frequency, we expect cap1 $\Delta \Delta C$. albicans to have a higher frequency of LOH after exposure to all hosts except for bli-3 hosts, which do not produce ROS. The cap $1 \Delta / \Delta$ strain had significantly higher host-associated LOH frequencies from all host backgrounds compared to in vitro (Fig. S3B). The cap1 $\Delta \Delta$ strain had significantly higher relative $\mathrm{LOH}$ frequency compared to wildtype $C$. albicans extracted from both healthy and sek-1 hosts (Fig. 4B). Unsurprisingly, we did not detect any significant differences in the relative LOH frequency between wildtype $C$. albicans and cap1 $\Delta \Delta C$. albicans extracted from bli-3 hosts which do not produce ROS(Fig. 4B). Together, these data indicate that host-produced ROS elevates $C$. albicans genome instability and cap $1 \mathrm{p}$ is important in detoxifying both in vivo and in vitro ROS.

Since ROS generated through the dual oxidase, BLI-3 causes genome instability in C. albicans, we wanted to determine whether antioxidants mitigate $C$. albicans genome instability from host-produced ROS. To test whether antioxidants reduce $C$. albicans genome instability caused by host-produced ROS, we measured $C$. albicans LOH frequency following host exposure with the addition of $25 \mu \mathrm{M}$ of lipoic acid ${ }^{37}$, a well research antioxidant involved in the breakdown of ROS. In C. albicans extracted from healthy hosts, we observed a significant reduction in relative LOH frequency with the addition of lipoic acid (Figs. 4C \& S5C). We observed a similar trend in $C$. albicans extracted from sek-1 hosts. Lipoic acid lowered the relative frequency of LOH in sek-1 hosts (Figs. 4C \& S5C). Following bli-3 host exposure $C$. albicans relative $\mathrm{LOH}$ frequency was not significantly different with or without lipoic acid (Figs. 4C \& S5C). This suggests that antioxidants only reduce $C$. albicans genome instability in hosts that produce ROS. Together, these data suggest that the host-produced ROS generates genome instability in C. albicans which can be alleviated by the use of antioxidants. 
A.

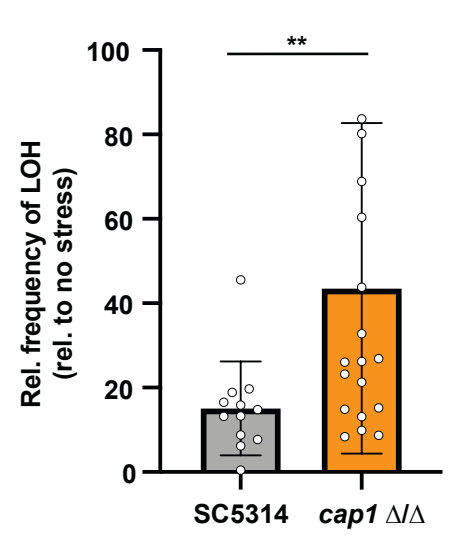

C.

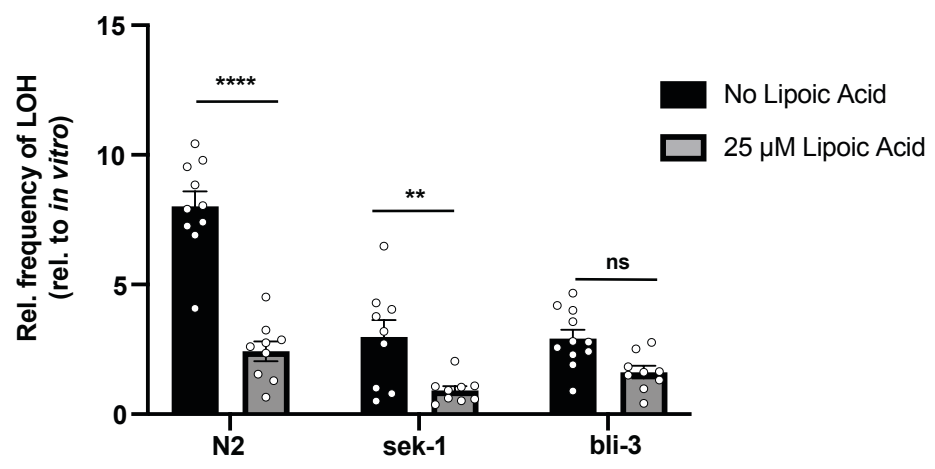

B.

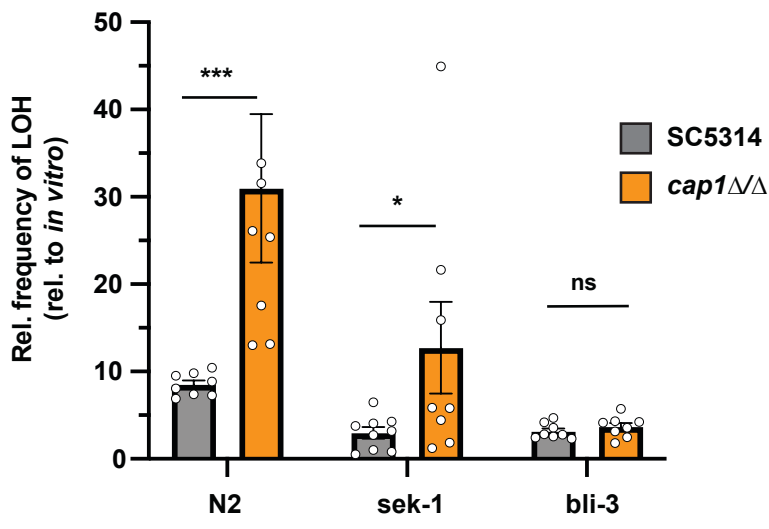

Figure 4: C. albicans cap1 $\Delta \Delta$ strain is more susceptible to in vitro and in vivo ROS

A) Plotted are the $\mathrm{LOH}$ frequencies of $C$. albicans exposed to $5 \mathrm{mM} \mathrm{H}_{2} \mathrm{O}_{2}$ for $24 \mathrm{~h}$ relative to the frequencies of $\mathrm{LOH}$ of $C$. albicans without stress exposure. Plotted are the means and SD for both SC5314 (grey, $\mathrm{n}=12$ ) and cap $1 \Delta / \Delta$ (orange, $\mathrm{n}=$

19). Each data point represents an individual measurement. B) Plotted are the $\mathrm{LOH}$ frequencies of $C$. albicans exposed to each host environment relative to the no host frequency of $\mathrm{LOH}$. Plotted are the means and SD. C) C. albicans LOH

frequency following host exposure relative to the no host LOH frequency with $25 \mu \mathrm{M}$ of alpha-lipoic acid (grey, N2: $\mathrm{n}=9$, sek-1: $\mathrm{n}=9$, bli-3: $\mathrm{n}=9$ ) and without $25 \mu \mathrm{M}$ of alpha-lipoic acid (black, N2: $\mathrm{n}=16$, sek-1: $\mathrm{n}=9$, bli-3: $\mathrm{n}=19$ ). Each data point represents an individual measurement. Plotted are the means and SD. Asterisks indicate significant differences $\left({ }^{* \star *} p\right.$ $<0.0001,{ }^{* * *} p<0.005,{ }^{* *} p<0.01,{ }^{*} p<0.05, n s=$ not significant; Mann-Whitney $U$ test $)$.

\section{Discussion}

We previously reported higher levels of genome instability in C. albicans exposed to wildtype hosts ${ }^{17}$. Here we investigated whether host innate immune pathways impact $C$. albicans genome stability. By using wildtype and two immunocompromised hosts with mutations in AMP production (sek-1) and ROS production (bli-3), we compared the differences in hostassociated $C$. albicans genome stability and mutational landscape. $C$. albicans associated with either immunocompromised host had reduced relative $\mathrm{LOH}$ frequencies compared to those associated with healthy hosts (Figure 1). Similar to several other host passaging experiments and whole-genome sequencing results from clinical isolates ${ }^{14,15,19}$, many of our isolates (5/6) extracted from wildtype hosts contained large-scale genomic changes including whole and segmental chromosomal aneuploidy and/or additional LOH events (Figure 2). Akin to Forche et. al., ${ }^{14}$ we detected the presence of an extra copy of chromosome 6 following host exposure. Their study suggests that this extra copy of chromosome 6 produces a more commensal-like phenotype inside the host environment. However, we have yet to investigate the virulence of our isolates. We also detected an extra copy of chromosome 7 in one isolate following wildtype host exposure, which in a gastrointestinal murine model of candidiasis is more fit in the gastrointestinal tract compared to the euploid parent ${ }^{15}$. Therefore, the generation of specific 
aneuploidies might enable host adaptation. However, removal of host-produced AMPs and ROS lead to the overall decrease in genetic variation. Immunocompromised host-associated $C$. albicans isolates did not carry any detectable aneuploidy and only a small number (2/8) had an $\mathrm{LOH}$ event that was not selected for (Figure 2). This suggests that both AMPs and ROS act as stressors on $C$. albicans that enable the generation of genetic variation which might lead to phenotypic changes that create a more synergistic host-pathogen relationship.

The host has a variety of mechanisms in order to control microbial growth. Because $C$. albicans is normally a commensal and resides in many niches in the human body, it must strike a delicate balance with the host to evade detection. Overgrowth of $C$. albicans initiates an immune response that initially includes the production of AMPs and the recruitment of phagocytes to the site of infection ${ }^{21}$. AMPs represent a potent defense mechanism designed to kill pathogens through a variety of mechanisms including disruption of the cell membrane and DNA damage ${ }^{23}$. Host pathways responsible for the production of AMPs including the $C$. elegans PMK-1 MAPK cascade represent an important conserved mechanism in controlling pathogen proliferation. Mutations in the C. elegans p38 ortholog, pmk-130 and the MKK3/6 ortholog, sek-1 lead to greater susceptibility to pathogens including C. albicans ${ }^{32,33}$. Our work demonstrates that removal of host AMPs decreases overall genetic diversity in $C$. albicans (Figures $1 \& 2$ ). This suggests that that the large-scale genomic changes generated in hosts with AMPs presents a strategy that enables $C$. albicans to survive this potent defense mechanism and evade future immune response.

Although counterintuitive, $\mathrm{LOH}$ increases genetic diversity by unmasking recessive alleles, leading to phenotypic changes ${ }^{41}$. For example, LOH of drug-resistant alleles of $E R G 11$, TAC1 or MRR1 increases antifungal drug resistance $42-44$. Similarly, aneuploidy offers a shortterm solution that organisms use during adaptation ${ }^{40,45,46}$ and have been shown to be advantages under certain conditions including the host environment ${ }^{14-16}$. Because we detect large-scale $\mathrm{LOH}$ events and aneuploidy in isolates exposed to healthy hosts, this is suggestive that these genetic changes could represent a mechanism for adaptation to the host.

In addition to the host environment increasing genetic diversity through $\mathrm{LOH}$ events, physiological stressors including ROS induce $\mathrm{LOH}$ and whole changes in ploidy ${ }^{25,28}$. Our work also demonstrated higher levels of genome instability in in vitro ROS (Fig. 4A). In addition, we found that $C$. albicans associated with bli-3 hosts unable to produce ROS, had lower LOH frequencies (Figure 1), and fewer genome-wide changes compared to wildtype hosts (Figure 2). Further, we also found that $C$. albicans had less INDELs following bli-3 host exposure (Figure $3 \mathrm{~B})$. These novel results suggest that the bli-3 host defense pathway generates genome instability and genetic variation in C. albicans. Recent work in E. coli, demonstrated that when exposed to low levels of ROS, E. coli exhibits a priming response in which evolution in ROS occurs faster and cells develop greater resistance as opposed to non-primed cells ${ }^{47}$. Our results suggest that host-produced ROS is priming $C$. albicans allowing for tolerance of greater stress.

C. albicans has several mechanisms for detoxify ROS, one of which is regulated by the transcription factor Cap1p. Cap1 $1 \Delta \Delta$ mutants are more susceptible to in vitro ROS killing ${ }^{48,49}$. However, no study has demonstrated this the impact of the Cap1p transcription factor for detoxifying ROS in vivo. Here we show that cap $1 \Delta \Delta C$. albicans genome instability is increased compared to wildtype C. albicans in hosts capable of producing ROS (N2 and sek-1) but not in bli-3 hosts incapable of producing ROS (Fig. 4B). Which suggests that host-produced ROS through the bli-3 dual-oxidase induces genome instability in C. albicans and that Cap $1 p$ is important for detoxification of host-produced ROS.

Exogenous antioxidants provide an alternative to the cellular mechanisms for detoxifying ROS. Adding antioxidants to Pre-SPO media increased cell viability and decreases genome instability in tetraploid C. albicans ${ }^{25}$ and prevented the oxidative stress dependent activation of Yap1p in Saccharomyces cerevisiae by lowering the overall redox potential ${ }^{50}$. To address if antioxidants reduced genome instability in our system, we exposed $C$. albicans to the well- 
studied antioxidant lipoic acid ${ }^{37}$ in healthy hosts and immunocompromised hosts. We found that lipoic acid reduced host-associated $C$. albicans genome instability in healthy and sek-1 hosts, but not bli-3 hosts (Fig. 4C). We propose that antioxidants reduce C. albicans genome instability only when they are exposed to host-produced ROS.

Our work identifies AMPs and ROS as important conserved innate immune responses that generate genome instability in the fungal pathogen, $C$. albicans. We propose that the generation of genetic variation in response to host-produce ROS and AMPs represents a way in which $C$. albicans can quickly respond to host stressors thus further tolerating these stressors or avoiding further immune attack. However, further studies are necessary to define the impact of host-generated genomic changes on the relationship between the host and the pathogen. Our work has important implications for opportunistic pathogens that are often commensal in healthy hosts but more frequently cause infection in immunocompromised hosts.

\section{Methods}

\section{Strains and Maintenance}

C. albicans and C. elegans strains for this study are listed in Table 1. Yeast strains were stored at $-80^{\circ} \mathrm{C}$ and maintained on YPD (yeast peptone dextrose; $1 \%$ yeast extract, $2 \%$ bactopeptone, $2 \%$ glucose, $0.004 \%$ adenine, $0.008 \%$ uridine) at $30^{\circ} \mathrm{C}$. Strains were initially struck onto YPD agar plates from frozen glycerol stocks and incubated at $30^{\circ} \mathrm{C}$ for 48 hours and single colonies used as the "parental strain" in subsequent in vivo experiments. Nematode populations were maintained on plates containing nematode growth media (NGM) with E. coli (OP50) for a food source. C. elegans were transferred to a new plate containing freshly seeded $E$. coli every three to four days. For genome stability assays, treatment plates were seeded with both $C$. albicans and $E$. coli and supplemented with $0.2 \mathrm{~g} / \mathrm{L}$ streptomycin to inhibit overgrowth of E. coli. For fecundity and genome stability assays, NGM was supplemented with $0.08 \mathrm{~g} / \mathrm{L}$ of uridine and $0.08 \mathrm{~g} / \mathrm{L}$ of histidine to facilitate growth of auxotrophic C. albicans strains.

\section{Host-associated C. albicans genome stability}

Host preparation: NGM plates are seeded with a mixture of E. coli and C. albicans $24 \mathrm{~h}$ prior to host preparation. To seed plates, single colonies of $C$. albicans were inoculated into 3 $\mathrm{mL}$ YPD and incubated overnight at $30^{\circ} \mathrm{C}$. Cultures were adjusted with $\mathrm{ddH}_{2} \mathrm{O}$ to a final concentration of $3.0 \mathrm{OD}_{600}$ per $\mathrm{mL}$. Simultaneously, a single colony of $E$. coli was inoculated into $50 \mathrm{~mL}$ LB and incubated for $24-48 \mathrm{~h}$ at $30^{\circ} \mathrm{C}$. The $E$. coli culture was pelleted and washed twice with $1 \mathrm{~mL} \mathrm{ddH} \mathrm{H}_{2} \mathrm{O}$. The pellet was weighed and diluted to final concentration of $200 \mathrm{mg} / \mathrm{mL}$. For in vitro treatments, $250 \mu \mathrm{L} \mathrm{C}$. albicans was spread onto $\mathrm{NGM}+$ streptomycin agar plates and incubated overnight at $30^{\circ} \mathrm{C}$. For in vivo treatment plates, $6.25 \mu \mathrm{L} \mathrm{C}$. albicans, $31.25 \mu \mathrm{L} \mathrm{E}$. coli, and brought to a final volume of $250 \mu \mathrm{L}$ with $\mathrm{ddH}_{2} \mathrm{O}$, was spread onto $\mathrm{NGM}+$ streptomycin agar plates and incubated overnight at $30^{\circ} \mathrm{C}$.

To synchronize $C$. elegans populations, nematodes and eggs were washed off plates with M9 buffer, transferred to $15 \mathrm{~mL}$ conical tubes, and pelleted via centrifugation (2 min at 1200 $\mathrm{rpm}$ ). The pellet was resuspended in $2 \mathrm{~mL}$ of $25 \%$ bleach, mixed via inversion for 2 minutes,

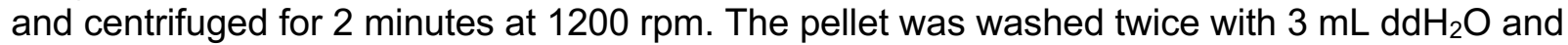

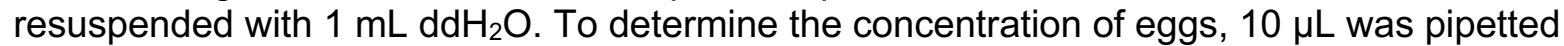
onto a concave slide, eggs were visualized microscopely and counted, and the suspension was adjusted to a concentration of $\sim 100$ eggs per $100 \mu \mathrm{L}$ with M9. 
Host-associated yeast extractions: Yeast extractions were performed as described previously ${ }^{17}$. Hosts exposed to $C$. albicans were washed from plates with $3 \mathrm{~mL} \mathrm{M9}$ and pelleted via centrifugation (2 min at 2,000 RPM). The supernatant was removed and the pellet was resuspended with $1 \mathrm{~mL} \mathrm{3 \%}$ bleach, transferred to a microcentrifuge tube, and incubated for three minutes and subsequently centrifuged for $1 \mathrm{~min}$ at 12,000 rpm. The supernatant was removed and washed with $1 \mathrm{~mL}$ of $\mathrm{M9}$ and centrifuged for one minute at 12,000 rpm. The wash was repeated two more times to ensure all bleach was removed. $100 \mu$ laliquots of nematode suspension were transferred to $0.6 \mathrm{~mL}$ clear microtubes for manual disruption with a motorized pestle. After one minute of manual disruption, the worm intestine solution was then diluted accordingly with an M9 buffer and plated on YPD $+0.034 \mathrm{mg} / \mathrm{L}$ chloramphenicol to select prevent any bacterial colonies from arising.

\section{GAL1 Loss of Heterozygosity assay}

In vitro: Single colonies of $C$. albicans were inoculated in $3 \mathrm{~mL}$ YPD grown overnight at $30^{\circ} \mathrm{C}$ and subsequently diluted to $3 \mathrm{OD}$ in $\mathrm{dd}_{2} \mathrm{O} .250 \mu \mathrm{L}$ was plated and spread onto NGM + streptomycin plates and incubated overnight at $30^{\circ} \mathrm{C}$ and transferred to $20^{\circ} \mathrm{C}$ for four days. On day four, yeast cells were washed off with $\mathrm{dd}_{2} \mathrm{O}$, harvested by centrifugation, washed once with $\mathrm{ddH}_{2} \mathrm{O}$, resuspended in $1 \mathrm{~mL}$ of $\mathrm{ddH}_{2} \mathrm{O}$ and serially diluted for single colony growth. To determine the total cell viability, $100 \mu \mathrm{L}$ of $10^{-6}$ dilution was plated onto YPD and grown for 48 hours at $30^{\circ} \mathrm{C}$. To identify cells that lost $G A L 1,100 \mu \mathrm{L}$ of $10^{-2}$ and $10^{-3}$ dilution was plated onto 2-deoxygalactose (2-DOG; $0.17 \%$ yeast nitrogen base without amino acids $0.5 \%$ ammonium sulfate, $0.0004 \%$ uridine, $0.0004 \%$ histidine, $0.1 \% 2$-deoxygalacose, $3 \%$ glycerol) and colony forming units (CFUs) counted following 72 hours incubation at $30^{\circ} \mathrm{C}$.

In vivo: The approach was very similar as the in vitro LOH assay described above, with the following changes. A population of $\sim 100$ nematodes were plated on each treatment plate containing both $C$. albicans and $E$. coli. On day four, yeast were extracted as described in the previous section. A dilution of $10^{-1}$ and $10^{-2}$ was plated on YPD + chloramphenicol to enumerate total growth and undiluted cells were plated on 2-DOG to select for the loss of GAL1. Three technical replicates were used for each $C$. albicans strain for both in vitro and in vivo experiments. At least three biological replicates were used for each genome stability assay.

\section{Lipoic Acid Experiments}

$\alpha$-Lipoic acid (Sigma-Aldrich \#1077-28-7) was dissolved in 100\% ethanol and added to NGM media containing $0.2 \mathrm{~g} / \mathrm{L}$ streptomycin sulfate to a final concentration of $25 \mu \mathrm{M}$. LOH assays were performed with $\alpha$-Lipoic acid as described above.

\section{Hydrogen Peroxide Exposure and Genome Stability:}

Single colonies of $C$. albicans were inoculated in either $2 \mathrm{ml}$ of YPD or in $2 \mathrm{ml}$ of YPD containing $5 \mathrm{mM} \mathrm{H}_{2} \mathrm{O}_{2}$, grown for $20 \mathrm{~h}$ at $30^{\circ} \mathrm{C}$. Cultures were centrifuged at $2000 \mathrm{rpm}$ for 2 minutes. The supernatant was removed, and the pellet was washed once with $1 \mathrm{ml}$ of $\mathrm{ddH}_{2} \mathrm{O}$. Cultures were serially diluted for single colony growth. Loss-of-heterozygosity assays were performed to determine the frequency of $\mathrm{LOH}$. Single colonies were randomly picked from YPD plates and genome size was assessed via flow cytometry.

\section{Whole genome sequencing and analysis}

Genomic DNA was isolated with phenol chloroform as described previously ${ }^{53}$. Whole genome sequencing was performed through the Microbial Genome Sequencing Center using a single library preparation method based on the Illumina Nextera kit. Libraries were sequencing 
using paired end $(2 \times 150 \mathrm{bp})$ reads on the NextSeq 550 platform. Adaptor sequences and lowquality reads were trimmed using Trimmomatic (v0.39 LEADING:3 TRAILING: 3

SLIDINGWINDOW: 4:15 MINLEN: 36 TOPHRED33) ${ }^{54}$. All reads were mapped to the phased $C$. albicans reference genome ${ }^{55}$ using the haplotypo python script 'mapping.py'. This tool uses the Burrows-Wheeler Aligner MEM (BWA v0.1.19) algorithm to align the sequencing reads to the reference genome followed by Samtools (v0.1.19) to sort, mark duplicates, and create a BAM file. Variant files were created using the haplotypo python script 'var_calling.py' using the BCFtools calling method.

Identification of aneuploidy, CNVs, and LOH were conducted using whole genome sequencing data and the Yeast Mapping Analysis Pipeline (YMAP). BAM files were uploaded to YMAP and plotted using the Candida albicans reference genome (A21-s02smo8-r09) with corrections for chromosome end bias and GC content ${ }^{56}$. Identification of SNPs, INDELs and Ts/TV: Variant files were analyzed using BCFtools 'stats' followed by plot-vcfstats to generate a visual representation of the proportion of each type of variant. PCA plot was made be generating GVCF files using haplotypo with the GATK call. To join all of the variant files, the CombineGVCFs GATK module (v.4.0.12.0) ${ }^{57}$ was used. Principle component analysis (PCA) of the SNPs was performed using SNPRelate ${ }^{58}$ in the R/Bioconductor Package.

Statistical analysis:

Statistical analysis was performed using GraphPad Prism 8 software. Data sets were tested for normality using the D'Agnostino \& Pearson omnibus normality test.

\section{Acknowledgments/Author Contributions:}

We would like to thank Dr. Levi Morran for critical reading of the manuscript. This research is supported by NSF DEB-1943415 (MAH) and Emory University startup funds (MAH).

ACS and MAH designed the study. ACS conducted all of the experiments. ACS and MAH analyzed the data. ACS and MAH wrote, reviewed and edited the manuscript. 


\section{References:}

1. Pfaller, M.A., Diekema, D.J. Epidemiology of Invasive Candidiasis: a Persistent Public Health Problem. Clinical Microbiology Reviews. 20 (1), 133-163, doi: 10.1128/cmr.00029-06 (2007).

2. Perlroth, J., Choi, B., Spellberg, B. Nosocomial fungal infections: epidemiology, diagnosis, and treatment. Medical Mycology. 45 (4), 321-346, doi: 10.1080/13693780701218689 (2007).

3. Sobel, J.D. Vulvovaginal candidosis. The Lancet. 369 (9577), 1961-1971, doi: 10.1016/s01406736(07)60917-9 (2007).

4. Akimoto-Gunther, L. et al. Highlights Regarding Host Predisposing Factors to Recurrent Vulvovaginal Candidiasis: Chronic Stress and Reduced Antioxidant Capacity. PLOS ONE. 11 (7), e0158870, doi: 10.1371/journal.pone.0158870 (2016).

5. Ahmad, A., Khan, A.U. Prevalence of Candida species and potential risk factors for vulvovaginal candidiasis in Aligarh, India. European Journal of Obstetrics \& Gynecology and Reproductive Biology. 144 (1), 68-71, doi: 10.1016/j.ejogrb.2008.12.020 (2009).

6. Grigoriou, O., Baka, S., Makrakis, E., Hassiakos, D., Kapparos, G., Kouskouni, E. Prevalence of clinical vaginal candidiasis in a university hospital and possible risk factors. European Journal of Obstetrics \& Gynecology and Reproductive Biology. 126 (1), 121-125, doi: 10.1016/j.ejogrb.2005.09.015 (2006).

7. Gonçalves, B., Ferreira, C., Alves, C.T., Henriques, M., Azeredo, J., Silva, S. Vulvovaginal candidiasis: Epidemiology, microbiology and risk factors. Critical Reviews in Microbiology. 42 (6), 1-23, doi: 10.3109/1040841x.2015.1091805 (2015).

8. Gunther, L.S.A., Martins, H.P.R., Gimenes, F., Abreu, A.L.P. de, Consolaro, M.E.L., Svidzinski, T.I.E. Prevalence of Candida albicans and non-albicans isolates from vaginal secretions: comparative evaluation of colonization, vaginal candidiasis and recurrent vaginal candidiasis in diabetic and non-diabetic women. Sao Paulo Medical Journal. 132 (2), 116-120, doi: 10.1590/1516-3180.2014.1322640 (2014).

9. Leon, E.M. de, Jacober, S.J., Sobel, J.D., Foxman, B. Prevalence and risk factors for vaginal Candidacolonization in women with type 1 and type 2 diabetes. BMC Infectious Diseases. 2 (1), 1, doi: 10.1186/1471-2334-2-1 (2002).

10. Iliev, I.D., Leonardi, I. Fungal dysbiosis: immunity and interactions at mucosal barriers. Nature Reviews Immunology. 17 (10), 635-646, doi: 10.1038/nri.2017.55 (2017).

11. Underhill, D.M., Iliev, I.D. The mycobiota: interactions between commensal fungi and the host immune system. Nature Reviews Immunology. 14 (6), nri3684, doi: 10.1038/nri3684 (2014).

12. Nucci, M., Anaissie, E. Revisiting the Source of Candidemia: Skin or Gut? Clinical Infectious Diseases. 33 (12), 1959-1967, doi: 10.1086/323759 (2001). 
13. Sitterlé, E., Maufrais, C., Sertour, N., Palayret, M., d'Enfert, C., Bougnoux, M.-E. Within-Host Genomic Diversity of Candida albicans in Healthy Carriers. Scientific reports. 9 (1), 2563, doi: 10.1038/s41598-019-38768-4 (2019).

14. Forche, A. et al. Rapid Phenotypic and Genotypic Diversification After Exposure to the Oral Host Niche inCandida albicans. Genetics. genetics.301019.2018, doi: 10.1534/genetics.118.301019 (2018).

15. Ene, I.V., Farrer, R.A., Hirakawa, M.P., Agwamba, K., Cuomo, C.A., Bennett, R.J. Global analysis of mutations driving microevolution of a heterozygous diploid fungal pathogen. Proceedings of the National Academy of Sciences. 115 (37), 201806002, doi: 10.1073/pnas.1806002115 (2018).

16. Forche, A. et al. Selection of Candida albicans trisomy during oropharyngeal infection results in a commensal-like phenotype. PLOS Genetics. 15 (5), e1008137, doi: 10.1371/journal.pgen.1008137 (2019).

17. Smith, A.C., Hickman, M.A. Host-Induced Genome Instability Rapidly Generates Phenotypic Variation across Candida albicans Strains and Ploidy States. mSphere. 5 (3), e00433-20, doi: 10.1128/msphere.00433-20 (2020).

18. Forche, A., Magee, P.T., Selmecki, A., Berman, J., May, G. Evolution in Candida albicans populations during a single passage through a mouse host. Genetics. 182 (3), 799-811, doi: 10.1534/genetics.109.103325 (2009).

19. Hirakawa, M.P. et al. Genetic and phenotypic intra-species variation in Candida albicans. Genome research. 25 (3), 413-25, doi: 10.1101/gr.174623.114 (2014).

20. Akira, S., Uematsu, S., Takeuchi, O. Pathogen Recognition and Innate Immunity. Cell. 124 (4), $783-$ 801, doi: 10.1016/j.cell.2006.02.015 (2006).

21. Netea, M.G., Brown, G.D., Kullberg, B.J., Gow, N.A.R. An integrated model of the recognition of Candida albicans by the innate immune system. Nature Reviews Microbiology. 6 (1), 67-78, doi: 10.1038/nrmicro1815 (2008).

22. Qin, Y. et al. Innate immune cell response upon Candida albicans infection. Virulence. 7 (5), 1-15, doi: 10.1080/21505594.2016.1138201 (2016).

23. Shai, Y. Mode of action of membrane active antimicrobial peptides. Peptide Science. 66 (4), 236-248, doi: 10.1002/bip.10260 (2002).

24. Jena, N.R. DNA damage by reactive species: Mechanisms, mutation and repair. Journal of Biosciences. 37 (3), 503-517, doi: 10.1007/s12038-012-9218-2 (2012).

25. Thomson, G.J., Hernon, C., Austriaco, N., Shapiro, R.S., Belenky, P., Bennett, R.J. Metabolisminduced oxidative stress and DNA damage selectively trigger genome instability in polyploid fungal cells. The EMBO journal. 38 (19), e101597, doi: 10.15252/embj.2019101597 (2019).

26. Basmaciyan, L., Bon, F., Paradis, T., Lapaquette, P., Dalle, F. "Candida Albicans Interactions With The Host: Crossing The Intestinal Epithelial Barrier.” Tissue Barriers. 7 (2), 1-31, doi: 10.1080/21688370.2019.1612661 (2019). 
27. Dantas, A. da S., Day, A., Ikeh, M., Kos, I., Achan, B., Quinn, J. Oxidative Stress Responses in the Human Fungal Pathogen, Candida albicans. Biomolecules. 5 (1), 142-165, doi: 10.3390/biom5010142 (2015).

28. Forche, A. et al. Stress Alters Rates and Types of Loss of Heterozygosity in Candida albicans. mBio. 2 (4), e00129-11, doi: 10.1128/mbio.00129-11 (2011).

29. Elkabti, A., Issi, L., Rao, R. Caenorhabditis elegans as a Model Host to Monitor the Candida Infection Processes. Journal of Fungi. 4 (4), 123, doi: 10.3390/jof4040123 (2018).

30. Kim, D.H. et al. A Conserved p38 MAP Kinase Pathway in Caenorhabditis elegans Innate Immunity. Science. 297 (5581), 623-626, doi: 10.1126/science.1073759 (2002).

31. Kim, D.H., Ausubel, F.M. Evolutionary perspectives on innate immunity from the study of Caenorhabditis elegans. Current Opinion in Immunology. 17 (1), 4-10, doi: 10.1016/j.coi.2004.11.007 (2005).

32. Feistel, D.J., Elmostafa, R., Nguyen, N., Penley, M., Morran, L., Hickman, M.A. A Novel Virulence Phenotype Rapidly Assesses Candida Fungal Pathogenesis in Healthy and Immunocompromised Caenorhabditis elegans Hosts. mSphere. 4 (2), doi: 10.1128/msphere.00697-18 (2019).

33. Feistel, D.J., Elmostafa, R., Hickman, M.A. Virulence phenotypes result from interactions between pathogen ploidy and genetic background. Ecology and Evolution. 10 (17), 9326-9338, doi:

10.1002/ece3.6619 (2020).

34. Moribe, H., Konakawa, R., Koga, D., Ushiki, T., Nakamura, K., Mekada, E. Tetraspanin Is Required for Generation of Reactive Oxygen Species by the Dual Oxidase System in Caenorhabditis elegans. PLoS Genetics. 8 (9), e1002957, doi: 10.1371/journal.pgen.1002957 (2012).

35. Hoeven, R. van der, McCallum, K.C., Cruz, M.R., Garsin, D.A. Ce-Duox1/BLI-3 Generated Reactive Oxygen Species Trigger Protective SKN-1 Activity via p38 MAPK Signaling during Infection in C. elegans. PLoS Pathogens. 7 (12), e1002453, doi: 10.1371/journal.ppat.1002453 (2011).

36. Hoeven, R. van der, Cruz, M.R., Chávez, V., Garsin, D.A. Localization of the Dual Oxidase BLI-3 and Characterization of Its NADPH Oxidase Domain during Infection of Caenorhabditis elegans. PLOS ONE. 10 (4), e0124091, doi: 10.1371/journal.pone.0124091 (2015).

37. Chávez, V., Mohri-Shiomi, A., Garsin, D.A. Ce-Duox1/BLI-3 Generates Reactive Oxygen Species as a Protective Innate Immune Mechanism in Caenorhabditis elegans ${ }^{\nabla}$. Infection and Immunity. 77 (11), 4983-4989, doi: 10.1128/iai.00627-09 (2009).

38. Jain, C., Pastor, K., Gonzalez, A.Y., Lorenz, M.C., Rao, R.P. The role of Candida albicans AP-1 protein against host derived ROS in in vivo models of infection. Virulence. 4 (1), 67-76, doi: $10.4161 /$ viru. 22700 (2013).

39. Irazoqui, J.E., Urbach, J.M., Ausubel, F.M. Evolution of host innate defence: insights from Caenorhabditis elegans and primitive invertebrates. Nature Reviews Immunology. 10 (1), 47-58, doi: 10.1038/nri2689 (2010). 
40. Yona, A.H. et al. Chromosomal duplication is a transient evolutionary solution to stress. Proceedings of the National Academy of Sciences. 109 (51), 21010-21015, doi: 10.1073/pnas.1211150109 (2012).

41. Feri, A. et al. Analysis of Repair Mechanisms following an Induced Double-Strand Break Uncovers Recessive Deleterious Alleles in the Candida albicans Diploid Genome. mBio. 7 (5), e01109-16, doi: 10.1128/mbio.01109-16 (2016).

42. Dunkel, N., Blaß, J., Rogers, P.D., Morschhäuser, J. Mutations in the multi-drug resistance regulator MRR1, followed by loss of heterozygosity, are the main cause of MDR1 overexpression in fluconazoleresistant Candida albicans strains. Molecular Microbiology. 69 (4), 827-840, doi: 10.1111/j.13652958.2008.06309.x (2008).

43. White, T.C. The presence of an R467K amino acid substitution and loss of allelic variation correlate with an azole-resistant lanosterol 14alpha demethylase in Candida albicans. Antimicrobial Agents and Chemotherapy. 41 (7), 1488-1494, doi: 10.1128/aac.41.7.1488 (1997).

44. Coste, A. et al. A Mutation in Tac1p, a Transcription Factor Regulating CDR1 and CDR2, Is Coupled With Loss of Heterozygosity at Chromosome 5 to Mediate Antifungal Resistance in Candida albicans. Genetics. 172 (4), 2139-2156, doi: 10.1534/genetics.105.054767 (2006).

45. Rancati, G. et al. Aneuploidy Underlies Rapid Adaptive Evolution of Yeast Cells Deprived of a Conserved Cytokinesis Motor. Cell. 135 (5), 879-893, doi: 10.1016/j.cell.2008.09.039 (2008).

46. Pavelka, N. et al. Aneuploidy confers quantitative proteome changes and phenotypic variation in budding yeast. Nature. 468 (7321), 321-325, doi: 10.1038/nature09529 (2010).

47. Rodríguez-Rojas, A. et al. Non-lethal exposure to $\mathrm{H} 2 \mathrm{O} 2$ boosts bacterial survival and evolvability against oxidative stress. PLOS Genetics. 16 (3), e1008649, doi: 10.1371/journal.pgen.1008649 (2020).

48. Enjalbert, B. et al. Role of the Hog1 Stress-activated Protein Kinase in the Global Transcriptional Response to Stress in the Fungal Pathogen Candida albicans. Molecular Biology of the Cell. 17 (2), 1018 1032, doi: 10.1091/mbc.e05-06-0501 (2006).

49. Alarco, A.-M., Raymond, M. The bZip Transcription Factor Cap1p Is Involved in Multidrug Resistance and Oxidative Stress Response inCandida albicans. Journal of Bacteriology. 181 (3), 700-708, doi: 10.1128/jb.181.3.700-708.1999 (1999).

50. Bednarska, S., Leroy, P., Zagulski, M., Bartosz, G. Efficacy of antioxidants in the yeast Saccharomyces cerevisiae correlates with their effects on protein thiols. Biochimie. 90 (10), 1476-1485, doi: 10.1016/j.biochi.2008.05.013 (2008).

51. Warris, A., Henriet, S.S.V. Invasive Fungal Infections in the Child with Chronic Granulomatous Disease. Current Fungal Infection Reports. 8 (1), 37-44, doi: 10.1007/s12281-013-0168-4 (2014).

52. Gerstein, A.C., Otto, S.P. Cryptic fitness advantage: diploids invade haploid populations despite lacking any apparent advantage as measured by standard fitness assays. PloS one. 6 (12), e26599, doi: 10.1371/journal.pone.0026599 (2011). 
53. Selmecki, A., Forche, A., Berman, J. Aneuploidy and Isochromosome Formation in Drug-Resistant Candida albicans. Science. 313 (5785), 367-370, doi: 10.1126/science.1128242 (2006).

54. Bolger, A.M., Lohse, M., Usadel, B. Trimmomatic: a flexible trimmer for Illumina sequence data. Bioinformatics. 30 (15), 2114-2120, doi: 10.1093/bioinformatics/btu170 (2014).

55. Muzzey, D., Schwartz, K., Weissman, J.S., Sherlock, G. Assembly of a phased diploid Candida albicansgenome facilitates allele-specific measurements and provides a simple model for repeat and indel structure. Genome Biology. 14 (9), R97, doi: 10.1186/gb-2013-14-9-r97 (2013).

56. Abbey, D.A. et al. YMAP: a pipeline for visualization of copy number variation and loss of heterozygosity in eukaryotic pathogens. Genome Medicine. 6 (11), 100, doi: 10.1186/s13073-014-0100-8 (2014).

57. McKenna, A. et al. The Genome Analysis Toolkit: A MapReduce framework for analyzing nextgeneration DNA sequencing data. Genome Research. 20 (9), 1297-1303, doi: 10.1101/gr.107524.110 (2010).

58. Zheng, X., Levine, D., Shen, J., Gogarten, S.M., Laurie, C., Weir, B.S. A high-performance computing toolset for relatedness and principal component analysis of SNP data. Bioinformatics. 28 (24), 3326-3328, doi: 10.1093/bioinformatics/bts606 (2012).

\section{Supplemental:}

\begin{tabular}{|c|c|c|}
\hline \multicolumn{3}{|c|}{ C. albicans strains } \\
\hline Strain & Genetic Background & Source \\
\hline YJB11700 & SC5314 reference strain, GAL1/gal $\triangle:: N A T$ & Gillum et. al. 1984; Hickman et al 2013 \\
\hline $\operatorname{cap} 1 \Delta \Delta$ & $\begin{array}{l}\text { his } 1 \Delta / \text { his } 1 \Delta, \text { leu2 } \Delta:: \text { C. dubliniensis HIS1/leu2 } \Delta:: \text { C. maltosa LEU2, } \arg 4 \Delta / \arg 4 \Delta \text {, } \\
\text { URA3/ura3 } \Delta:: \text { imm434, IRO1/iro1 } \Delta:: \text { imm434 } \\
\text { orf19.1623 }:: \text { C. dubliniensisHIS1/orf19.1623 } \Delta:: \text { C. maltosaLEU2 }\end{array}$ & Nobel et al 2010 \\
\hline \multicolumn{2}{|r|}{ C. elegans strains } & \\
\hline Alias & Genotype & \\
\hline WT & N2 (bristol) & \\
\hline bli-3 & bli-3(e767) & \\
\hline sek-1 & sek-1(km4) & \\
\hline
\end{tabular}

Table S1: List of strains used in this study 
A.

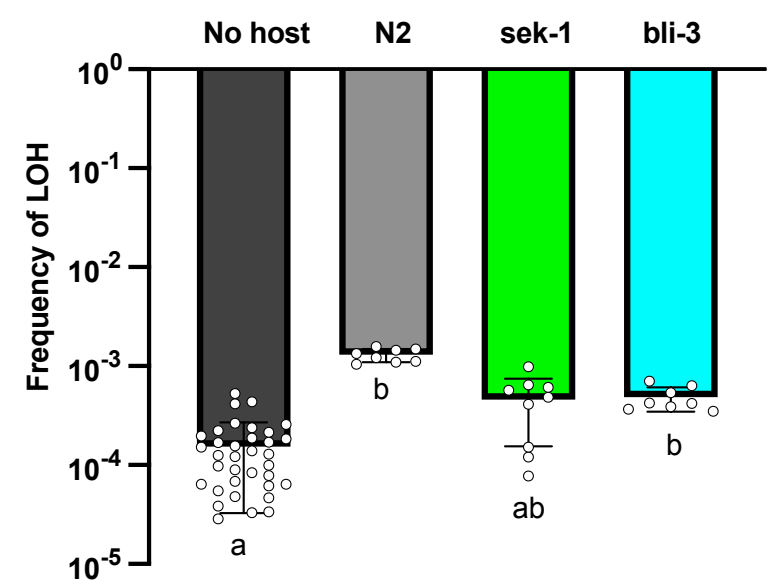

B.

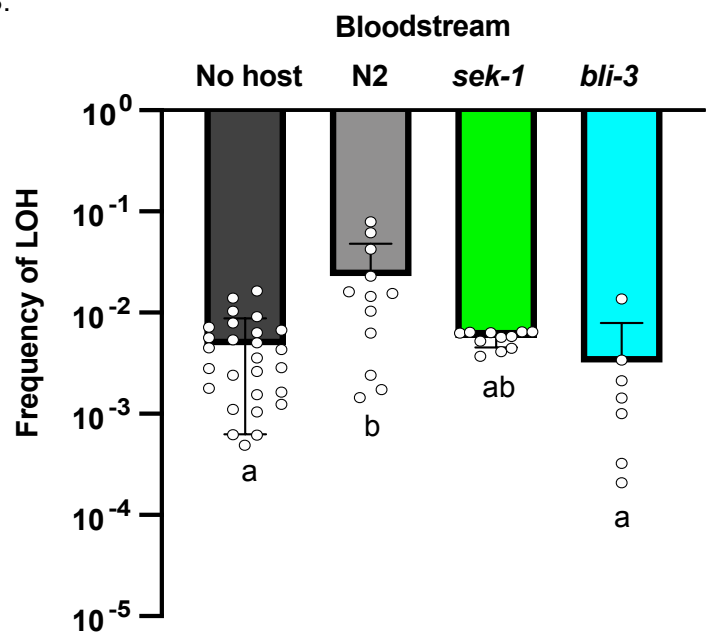

Figure S1: A) No host (dark grey, $\mathrm{n}=35$ ) and host-associated GAL1 LOH frequencies for laboratory $C$. albicans extracted from wildtype (N2) (grey, $n=8$ ), sek-1 (green, $n=9$ ), and bli-3 hosts (blue, $n=8$ ). Plotted are the mean and standard deviation. Symbols represent individual measurements. B) No host (dark grey, $n=27$ ) and host-associated $G A L 1 \mathrm{LOH}$ frequencies for a clinical bloodstream strain of $C$. albicans extracted from wildtype (N2) (grey, $n=12$ ), sek-1 (green, $\mathrm{n}=11$ ), and bli-3 hosts (blue, $\mathrm{n}=7$ ). Plotted are the mean and standard deviation. Symbols represent individual measurements. Treatments that share letters are not significantly different, whereas treatments with different letters are statistically different according to a Kruskal-Wallis test with post hoc Dunn's multiple comparisons $(p<0.05)$. 
A.

\author{
Non-LOH isolates
}

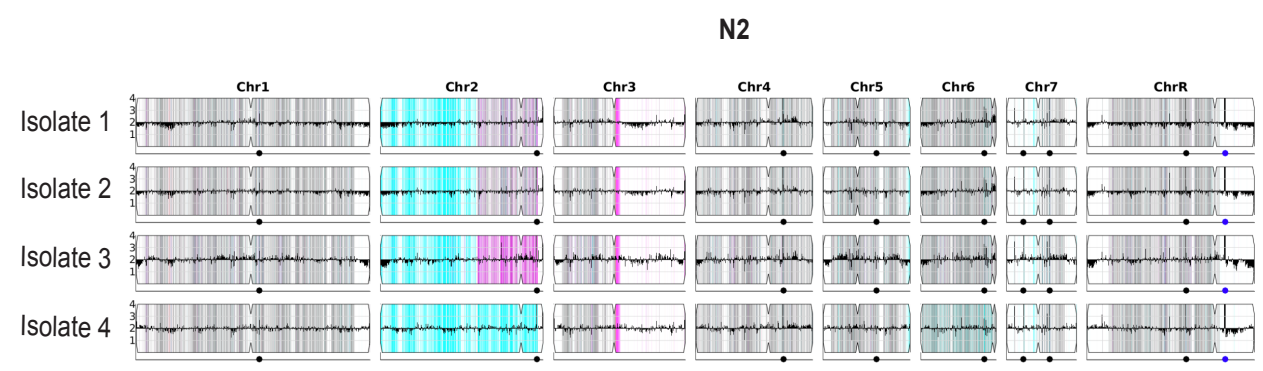

sek-1

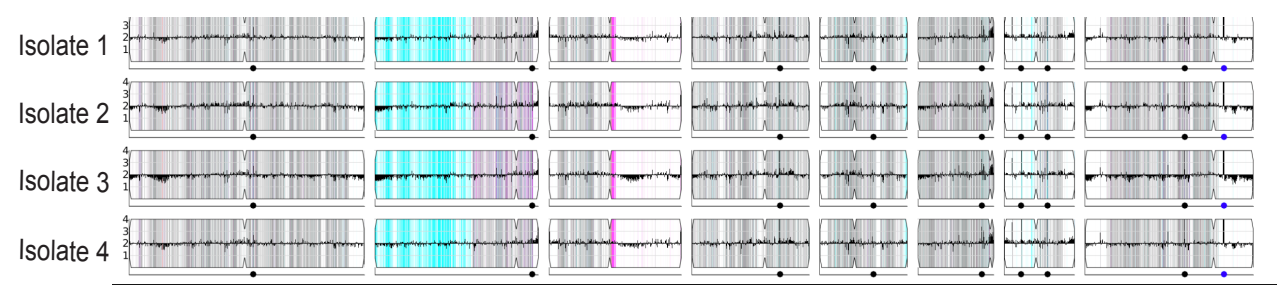

B.

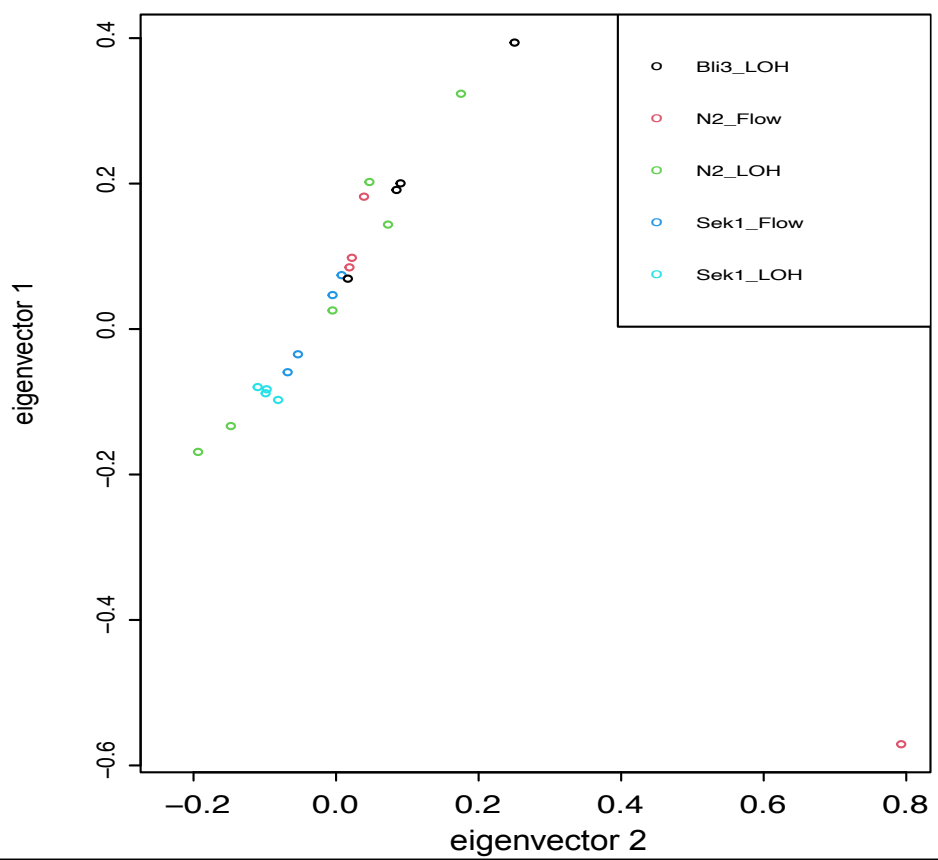

Figure S2: A) Following exposure to N2 (wildtype) and sek-1 C. albicans was extracted and colonies were chosen at random for DNA extraction and whole-genome sequencing. YMAPs showing the copy number and allelic ratio for each isolate following host exposure. B) Eigenvector plot displaying the top two eigenvectors (7.7\% and $7.2 \%)$ 
A.

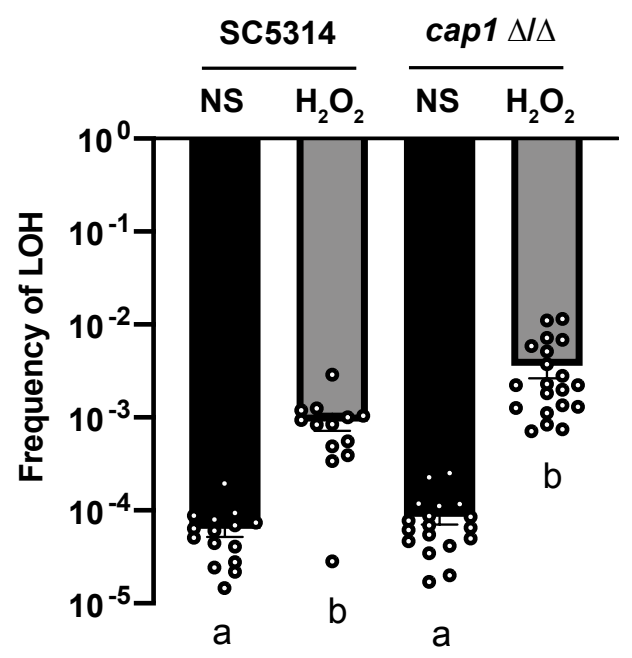

C.

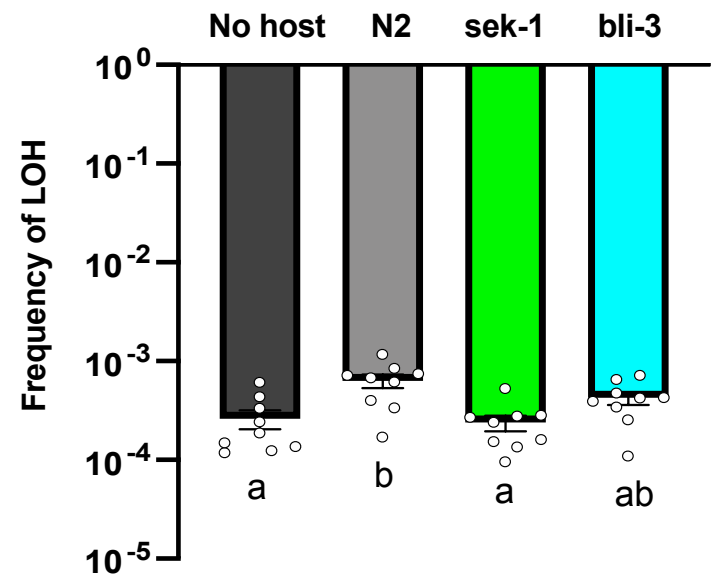

B. $\operatorname{cap} 1 \mathrm{~N} \Delta$

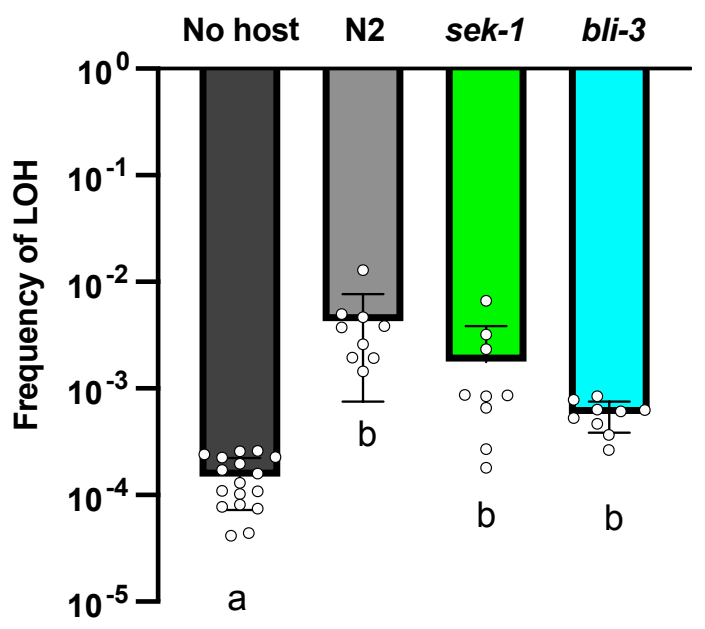

Figure S3: A) A) Plotted are the $\mathrm{LOH}$ frequencies $C$. albicans exposed to $5 \mathrm{mM} \mathrm{H}_{2} \mathrm{O}_{2}$ or no stress for $24 \mathrm{~h}$. Plotted are the means and SD for both SC5314 (no stress: black, $\mathrm{n}=15 ; \mathrm{H}_{2} \mathrm{O}_{2}$ : grey, $\mathrm{n}=13$ ) and cap1 $\Delta / \Delta$ (no stress: black, $\mathrm{n}=18 ; \mathrm{H}_{2} \mathrm{O}_{2}$ : grey, $\mathrm{n}=20$ ). Each data point represents an individual measurement. B) No host (dark grey, $n=17$ ) and host-associated GAL1 LOH frequencies for C. albicans extracted from wildtype (N2) (grey, $n=9$ ), sek-1 (green, $n$ $=9$ ), and bli-3 hosts (blue, $n=9$ ). Plotted are the mean and standard deviation. Symbols represent individual measurements. B) No host (dark grey, $n=9$ ) and host-associated GAL1 LOH frequencies for a $C$. albicans extracted from wildtype (N2) (grey, $n=9$ ), sek-1 (green, $n=$ 9), and bli-3 hosts (blue, $n=9$ ) with $25 \mu \mathrm{M}$ of alpha-lipoic acid. Plotted are the mean and SD. Symbols represent individual measurements. Treatments that share letters are not significantly different, whereas treatments with different letters are statistically different according to a Kruskal-Wallis test with post hoc Dunn's multiple comparisons $(p<0.05)$. 